\title{
On the Ternary Approach to Clifford Structures and Ising Lattices
}

\author{
J. Ławrynowicz, O. Suzuki and A. Niemczynowicz
}

\author{
In memory of Professor Jaime Keller
}

\begin{abstract}
We continue to modify and simplify the Ising-Onsager-Zhang procedure for analyzing simple orthorhombic Ising lattices by considering some fractal structures in connection with Jordan and Clifford algebras and by following Jordan-von Neumann-Wigner (JNW) approach. We concentrate on duality of complete and perfect JNW-systems, in particular ternary systems, analyze algebras of complete JNW-systems, and prove that in the case of a composition algebra we have a self-dual perfect JNW-system related to quaternion or octonion algebras. In this context, we are interested in the product table of the sedenion algebra.
\end{abstract}

Mathematics Subject Classification (2010). Primary 82C44; Secondary 82D25, 81R05, 15A66.

Keywords. Clifford algebra, crystal lattice, Ising lattice, Jordan algebra, octonions, quaternions, sedenions.

\section{Introduction and the organization of the paper}

After recalling Jordan-von-Neumann-Wigner (JNW) procedure and IsingOnsager-Zhang (IOZ) approach, we discuss a JNW-system and related fractal structures. Then, we turn our attention to the duality structure of complete and perfect ternary systems. We consider algebras of JNW-systems, in particular algebras of Clifford type. We conclude with a study of a composition algebra and self-dual perfect JNW-systems. Continuing our previous papers $[10,11,13]$ we prove in this case it is one of quaternion and octonion algebras, and this needs a study of the product table of sedenion algebra. The idea of applying the JNW-systems, considered in Section 3 and thereafter, to binary and ternary alloys may be to some extent illustrated for instance by the first step of fractal construction related to an $A B_{3}$ binary alloy of an fcc lattice and (111) surface orientation [2, Fig. 1]. 

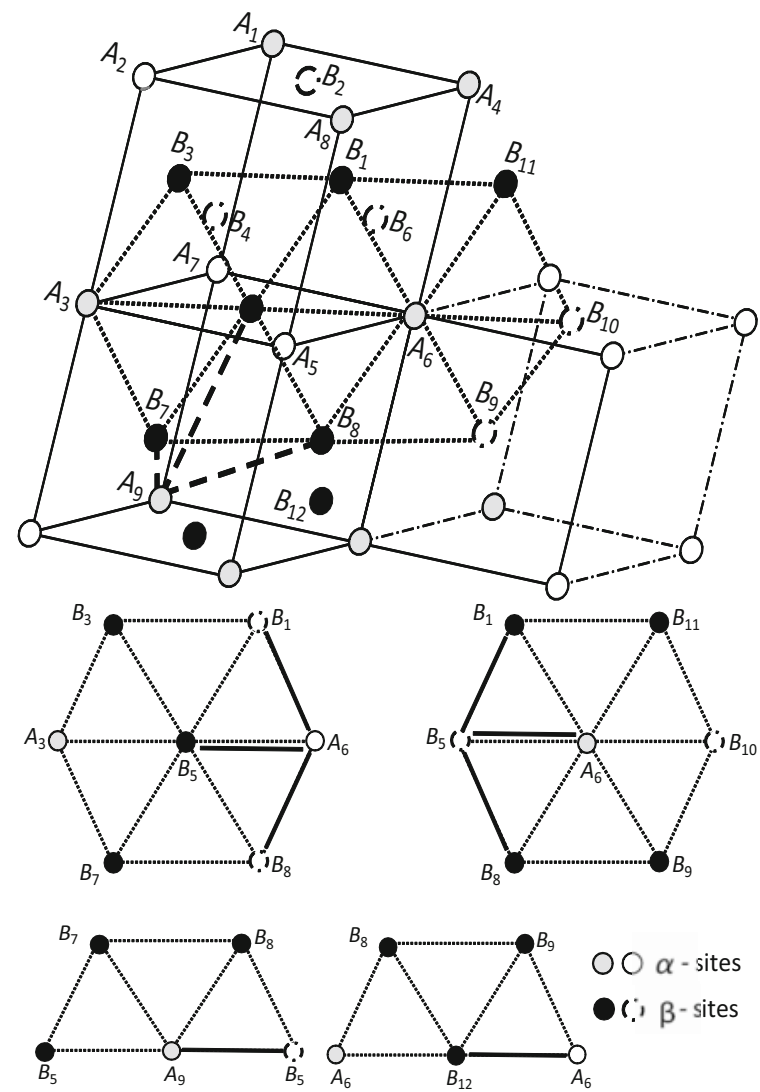

Figure 1. $A B_{3}$ binary alloy of an fcc lattice and (111) surface orientation.

The choice in this first step of the fractal construction is tightly related to the theory of cooperative phenomena of Kikuchi [9]. From our point of view it is important that like in the case of modern building model constructions, Kikuchi takes into account collections of adjacent edges, mostly three or four, of the crystal lattice. This suggests our subsequent steps: duality of fractals, duality fractals, JNW-systems, duality structure of complete JNW-systems, composition algebras of self-dual perfect JNW-systems, etc. These ideas can also be expressed by the following scheme:

JNW-systems

fractals including their duality $\longleftrightarrow$ binary and ternary alloys 
The content of the paper may be visualized as follows:

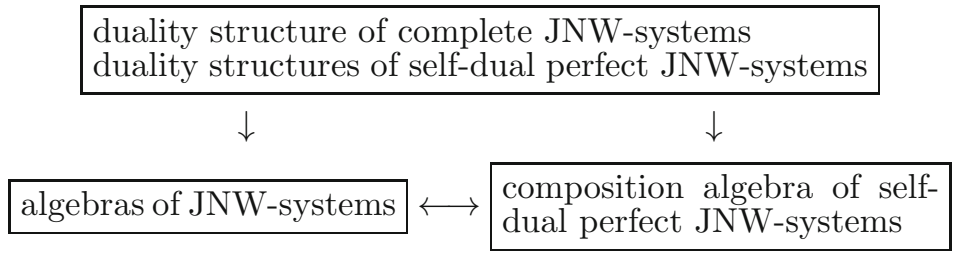

In order to pass from (1.1) to (1.2), we need the following expository (historical) part of the paper:

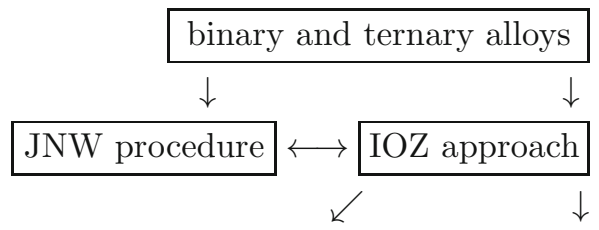

\begin{tabular}{l}
$\begin{array}{l}\text { JNW elements, complete elements, per- } \\
\text { fect elements }\end{array} \begin{array}{l}\text { fractal structure of } \\
\text { JNW procedure }\end{array}$ \\
$\qquad \begin{array}{ll}\downarrow & \downarrow \\
\text { construction of perfect } \\
\text { JNW-systems }\end{array}$ \\
\hline
\end{tabular}

\section{Jordan-von Neumann-Wigner procedure}

Jordan, von Neumann, and Wigner proved [7] (cf. also [4-6]) that among irreducible algebras there are algebras $\mathbb{H}_{q}(\mathbb{F})$ of Hermitian $q \times q$-matrices with entries in a field $\mathbb{F}$, namely

$$
\mathbb{H}_{3}(\mathbb{O}) \text { and } \mathbb{H}_{q}(\mathbb{F}) \text { with } \mathbb{F}=\mathbb{R}, \mathbb{C}, \mathbb{H} \text { and } q>2 \text {. }
$$

As it was observed in [11, p. 737], and then repeated in [21, p. 7], the natural appearance of multiplication

$$
A \circ B=\frac{1}{2}(A B+B A)
$$

instead of the usual matrix multiplication replaces in elegant way the desire of Ising [3], Onsager [16], Zhang [20], and Staples [18] of finding commutative subalgebras of the given algebra and studying their properties related to the superposition of bilinear forms. 


\section{Ising-Onsager-Zhang approach}

The models of Ising [3] and Onsager [16] are extended by Zhang [20] so that the resulting model applies to a simple orthorhombic crystal lattice in a threedimensional space. This extension involves, in particular, opening knots by a rotation in a higher dimensional space, and studying important commutators in the corresponding algebra.

In our approach, we extend Zhang's idea of decomposing knots related to lattice vertices to deformations of formal knot sequences arising from Reidemeister moves [19]. The model consisting of an orthombic lattice constructed by $m$ rows and $n$ sites per row in one of the $\ell$ planes involves [11] $2^{n \cdot \ell_{\text {}}}$ dimensional matrices $s_{r, s}^{\alpha}, \alpha=1,2,3,1 \leq s \leq \ell, 1 \leq r \leq n$, related to the so-called split-quaternions and, correspondingly, to the familiar Pauli matrices

$$
\sigma_{1}=\left(\begin{array}{ll}
0 & 1 \\
1 & 0
\end{array}\right), \quad \sigma_{2}=\left(\begin{array}{cc}
0 & -i \\
i & 0
\end{array}\right), \quad \sigma_{3}=\left(\begin{array}{cc}
1 & 0 \\
0 & -1
\end{array}\right)
$$

namely:

$$
\begin{aligned}
& s_{r, s}^{1}=\mathbf{1} \otimes \mathbf{1} \otimes \cdots \otimes \mathbf{1} \otimes \mathbf{1} \otimes \sigma_{1} \otimes \mathbf{1} \otimes \cdots \otimes \mathbf{1} \otimes \mathbf{1}, \\
& s_{r, s}^{2}=\mathbf{1} \otimes \mathbf{1} \otimes \cdots \otimes \mathbf{1} \otimes \mathbf{1} \otimes \frac{1}{i} \sigma_{2} \otimes \mathbf{1} \otimes \cdots \otimes \mathbf{1} \otimes \mathbf{1} \quad \text { with } \quad \mathbf{1}=\left(\begin{array}{ll}
1 & 0 \\
0 & 1
\end{array}\right), \\
& s_{r, s}^{3}=\mathbf{1} \otimes \underbrace{\mathbf{1} \otimes \cdots \otimes \mathbf{1}}_{r-2, s-2} \otimes \sigma_{3} \otimes \underbrace{\mathbf{1} \otimes \cdots \otimes \mathbf{1}}_{n-r, \ell-s} .
\end{aligned}
$$

It is worthwhile to notice that both Onsager and Zhang analyze continued fractions and nested series, e.g.,

$$
\frac{\sqrt{5}-1}{2}=\frac{1}{1+\frac{1}{1+\frac{1}{1+\frac{1}{1+\ldots}}}}=\sqrt{1-\sqrt{1-\sqrt{1-\sqrt{1-\sqrt{1-\ldots}}}}}
$$

which give rise to two kinds of fractals: of flower type and of branch type, and to the problem of their duality $[8,10]$. The objects of type (3.1) appear as a result of calculations in [16] and [20], but they inspire in us a relationship with the two types of fractals mentioned.

\section{Jordan-von Neumann-Wigner system}

We begin by introducing two kinds of systems for a set

$$
\left\{e_{1}, e_{2}, \ldots, e_{n}\right\} \text {. }
$$

The first system is called a triad. It is obtained by choosing three distinct elements from the set (4.1), for example, $\left(e_{1}, e_{2}, e_{3}\right)$. The second system is our JNW-system: we choose two elements, for example, $\left(e_{i}, e_{j}\right)$. Then, we select the third element, for example, $e_{k} \notin\left\{e_{i}, e_{j}\right\}$, and by doing so we define a JNW-element: the triple $\left(e_{i}, e_{j}, e_{k}\right)$. The most important elements arise from 
the binary product of an algebra. All JNW-elements selected from (4.1) make up a JNW-system.

Definition 4.1. A JNW-system consisting exclusively of complete JNW-elements is called complete if it satisfies the following conditions:

(a) For any pair of elements $\left(e_{i}, e_{j}\right)$ we can find a unique element $e_{k}$ which constitutes a JNW-element $\left(e_{i}, e_{j}, e_{k}\right)$.

(b) For any element $e_{k}$ we can find a (not necessarily unique) pair $\left(e_{i}, e_{j}\right)$ which constitutes a JNW-element $\left(e_{i}, e_{j}, e_{k}\right)$.

(c) Each element is symmetric, i.e.,

$$
\left(e_{i}, e_{j}, e_{k}\right)=\left(e_{\sigma(i)}, e_{\sigma(j)}, e_{\sigma(k)}\right)
$$

for any permutation $\sigma$ of the three objects considered.

Definition 4.2. A JNW-system consisting exclusively of perfect JNW-elements is called perfect if it satisfies conditions (a)-(c) from Definition 4.1 with the condition (b) replaced by:

(d) For any element $e_{k}$ we can find pairs $\left(e_{i}, e_{j}\right)$ such that each triple $\left(e_{i}, e_{j}, e_{k}\right)$ is a JNW-element and where the number of pairs $\left(e_{i}, e_{j}\right)$ does not depend on the given $e_{k}$.

\section{Fractal structure of Jordan-von Neumann-Wigner procedure}

We recall the following result from [11].

Theorem A. Let $\left(e_{1}, e_{2}, \ldots, e_{n}\right)$ be an $n$-sequence.

(A1) The sequence generates a complete JNW-system if and only if $n=$ $(2 l+1) 2^{k}-1, l=1,2, \ldots, k=2,3, \ldots ;$

(A2) The sequence generates a perfect JNW-system if and only if $n=2^{k}-$ $1, k=2,3, \ldots$.

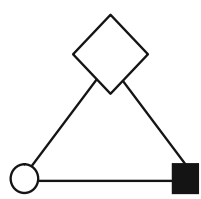

(a)

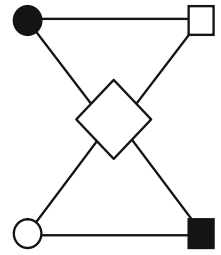

(b)

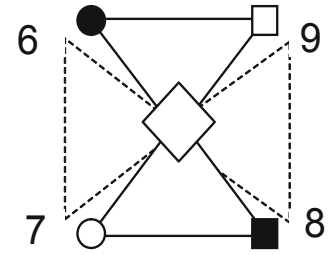

(c)

Figure 2. An example of construction of simple complete JNW-systems. 


\section{Construction of a perfect JNW-system}

As in [11], we construct a base system of ternaries $Z(n)$ and generate the total system. For $n=3$ we take $\left(e_{1}, e_{2}, e_{3}\right)$ and let $Z(3)=(1,2,3)$. Next, we choose one element from among $e_{1}, e_{2}, e_{3}$ which we call a polar point. Here, for example, we choose $e_{1}$, therefore we consider elements $\left(e_{1}, e_{2}, e_{3}\right),\left(e_{1}, e_{4}, e_{6}\right)$ and $\left(e_{1}, e_{5}, e_{7}\right)$. The later step, involving $e_{4}, e_{5}, \ldots$ does not apply in the case of $n=3$.

Then, we fix the JNW-system:

$$
Z(15)=(2,3) *_{1}(4,6) *_{1}(5,7) *(8,10) *_{1}(9,11) *_{1}(12,14) *_{1}(13,15) .
$$

The relation $*_{1}$, connected with the vertex $e_{1}$, is visualized in Fig. 3 .

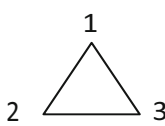

(a)

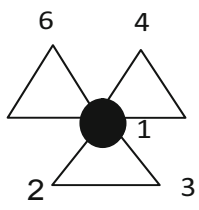

(b)

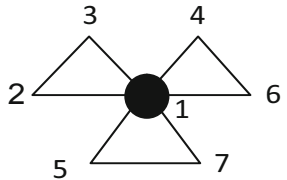

5

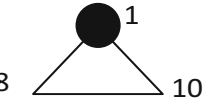

(c)

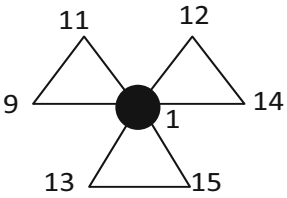

13

Figure 3. An example of construction of simple perfect JNW-systems.

Repeating this process and making a network, understood as a connected system of non intersecting triangles, such that each pair of neighboring element has a common vertex or common site, we can obtain a complete set of all perfect JNW-systems, in question.

It is clear that the structure in question is perfectly suitable for investigating ternary alloys without vacancies, binary, as well as ternary alloys with vacancies (cf. the triangle $\triangle(1,8,10)$ in Fig. $3(\mathrm{c}))$.

\section{Duality structure of complete JNW-systems and the characterization of self-dual perfect JNW-systems}

In this section we shall determine a duality structure for complete ternary systems and introduce a concept of a self-dual perfect JWN-system which characterizes the composition algebra directly. At first, we describe the duality structure for a complete JNW-system. 


\subsection{The duality structure of complete ternary systems}

We discuss the duality structure of complete ternary systems. By use of the basic construction of complete ternary systems, let us define

$$
\Lambda: \Lambda(1)=1, \quad \Lambda(t)=2 t, 2 \leq t \leq 2^{k-1} .
$$

Hence, we have a mapping called a duality mapping of genetic type, cf. [12]. We refer to each of the following three sets

$$
\{1\}, \quad\left\{t: 2 \leq t \leq 2^{k-1}\right\}, \quad\left\{\Lambda(t): 2 \leq t \leq 2^{k}\right\}
$$

as a branch of the system. A ternary system is called self dual when each branch of the system is preserved under the duality mapping.

\subsection{The dual structure of perfect ternary systems}

We are going to discuss the duality structure of perfect ternary systems:

$$
\Lambda: \Lambda(2)=2, \quad \Lambda\left(2^{k-1}\right)=2^{k-1}+2, k>2 ; \quad \Lambda(t)=2 t, 3 \leq t<2^{k-1} .
$$

Hence, we have the mapping which is natural to call duality of JNW-type. Moreover, we have the following theorem.

\section{Theorem 7.1.}

(T1) Each complete JNW-system is self dual.

(T2) A perfect JNW-system is self dual if and only if $n=3$ or $n=7$.

\section{Algebras of JNW-systems}

We are going to introduce algebras of a JNW-system. Here we restrict ourselves to a special class of the following unital real (not necessarily associative) algebras.

Definition 8.1. A finitely generated algebra $A=\mathbb{R}\left[e_{1}, e_{2}, \ldots, e_{n}\right]$ is said to be of monic product type if, for any two generators $e_{i}, e_{j} \in A$, we have $e_{i} e_{j}=\eta_{i j} e_{k}$ for some generator $e_{k}$ and $\eta_{i j} \in \mathbb{R}$.

We concentrate on algebras of a perfect JNW-system. We begin with the following definition.

Definition 8.2. A finitely generated algebra $A=\mathbb{R}\left[e_{1}, e_{2}, \ldots, e_{n}\right]$ is said to be of Clifford type if the following commutation relations are satisfied:

$$
e_{i} e_{j}+e_{j} e_{i}=0 \text { for all } i \neq j \text { and } i, j \neq 1 \text {. }
$$

In the case when $A$ is an associative, it becomes a Clifford algebra when it additionally satisfies the following condition:

$$
e_{j}^{2}=\eta_{j} e_{i} \quad \text { for some } i \text { and } \eta_{j} \in \mathbb{R} .
$$

The following theorem has been proven in [11]: 


\section{Theorem B.}

(B1) Let $A$ be a finitely generated algebra $A=\mathbb{R}\left[e_{1}, e_{2}, \ldots, e_{n}\right]$ of Clifford type. Then the JNW-system of $A$ is perfect.

(B2) Conversely, choosing a perfect system, we can find an algebra of Clifford type which realizes the system.

We illustrate Theorem B with a few examples.

Example 1. Mendel algebra:

\begin{tabular}{r|rr} 
& $e_{1}$ & $e_{2}$ \\
\hline$e_{1}$ & $2 e_{1}$ & $e_{2}$ \\
$e_{2}$ & $e_{2}$ & 0
\end{tabular}

Example 2. Algebra of complex numbers:

\begin{tabular}{r|rr} 
& $e_{1}$ & $e_{2}$ \\
\hline$e_{1}$ & $e_{1}$ & $e_{2}$ \\
$e_{2}$ & $e_{2}$ & $-e_{1}$
\end{tabular}

Example 3. Quaternion algebra:

\begin{tabular}{r|rrrr} 
& $e_{1}$ & $e_{2}$ & $e_{3}$ & $e_{4}$ \\
\hline$e_{1}$ & $e_{1}$ & $e_{2}$ & $e_{3}$ & $e_{4}$ \\
$e_{2}$ & $e_{2}$ & $-e_{1}$ & $e_{4}$ & $-e_{3}$ \\
$e_{3}$ & $e_{3}$ & $-e_{4}$ & $-e_{1}$ & $e_{2}$ \\
$e_{4}$ & $e_{4}$ & $e_{3}$ & $-e_{2}$ & $-e_{1}$
\end{tabular}

Example 4. Octonion algebra ( $\varepsilon=1$ in the table below):

\begin{tabular}{r|rrrrrrrr} 
& $e_{0}$ & $e_{1}$ & $e_{2}$ & $e_{3}$ & $e_{4}$ & $e_{5}$ & $e_{6}$ & $e_{7}$ \\
\hline$e_{0}$ & $e_{0}$ & $e_{1}$ & $e_{2}$ & $e_{3}$ & $e_{4}$ & $e_{5}$ & $e_{6}$ & $e_{7}$ \\
$e_{1}$ & $e_{1}$ & $-e_{0}$ & $e_{3}$ & $-e_{2}$ & $e_{5}$ & $-e_{4}$ & $-\varepsilon e_{7}$ & $\varepsilon e_{6}$ \\
$e_{2}$ & $e_{2}$ & $-e_{3}$ & $-e_{0}$ & $e_{1}$ & $e_{6}$ & $\varepsilon e_{7}$ & $-e_{4}$ & $-\varepsilon e_{5}$ \\
$e_{3}$ & $e_{3}$ & $e_{2}$ & $-e_{1}$ & $-e_{0}$ & $e_{7}$ & $-\varepsilon e_{6}$ & $\varepsilon e_{5}$ & $-e_{4}$ \\
$e_{4}$ & $e_{4}$ & $-e_{5}$ & $-e_{6}$ & $-e_{7}$ & $-e_{0}$ & $\varepsilon e_{1}$ & $\varepsilon e_{2}$ & $e_{3}$ \\
$e_{5}$ & $e_{5}$ & $e_{4}$ & $-\varepsilon e_{7}$ & $-e_{6}$ & $-\varepsilon e_{1}$ & $-e_{0}$ & $-\varepsilon e_{3}$ & $\varepsilon e_{2}$ \\
$e_{6}$ & $e_{6}$ & $\varepsilon e_{7}$ & $e_{4}$ & $-\varepsilon e_{5}$ & $-\varepsilon e_{2}$ & $\varepsilon e_{3}$ & $-e_{0}$ & $-\varepsilon e_{1}$ \\
$e_{7}$ & $e_{7}$ & $-\varepsilon e_{6}$ & $e_{5}$ & $e_{4}$ & $-e_{3}$ & $-\varepsilon e_{2}$ & $\varepsilon e_{1}$ & $-e_{0}$
\end{tabular}

Example 5. See the sedenion algebra defined in Table 5.

\section{Composition algebra and self-dual perfect JNW-systems}

Finally, we characterize composition algebras in terms of the JNW-systems. At first, we recall the definition of a composition algebra.

Definition 9.1. A unital algebra $A$ over $\mathbb{R}$ with a nondegenerate norm $N$ : $A \rightarrow \mathbb{R}^{+}$is a composition algebra if $N(X Y)=N(X) N(Y)$ for any two elements $X, Y \in A$. 


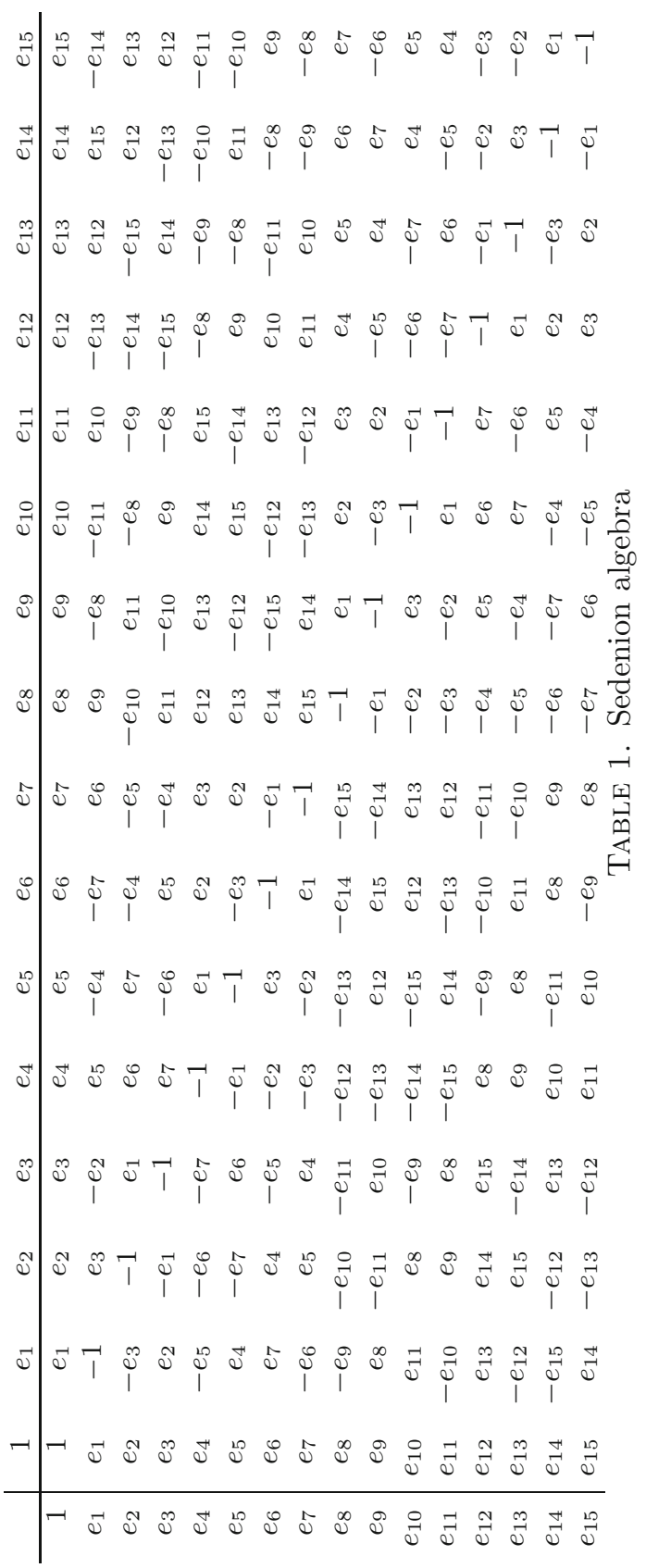


Definition 9.2. An algebra $A$ with a norm $N: A \rightarrow \mathbb{R}^{+}$is said to be ternary of composition type if its two ternaries $\left(e_{i}, e_{j}, e_{k}\right)$ and $\left(e_{r}, e_{s}, e_{t}\right)$ have a common vertex, for example $e_{k}=e_{t}$. When studying the commutation relations we may use the notation $\left(e_{i}, e_{j}: e_{k}\right)$, etc..

We know from the Hurtwitz's Theorem that every (unital) composition algebra (over the reals) which, at the same time, is a normed division algebra, is isomorphic to one of the following four algebras: the real numbers $\mathbb{R}$, the complex numbers $\mathbb{C}$, the quaternions $\mathbb{H}$, or the Cayley numbers (the octonions) $\mathbb{O}$.

Here we shall treat this characterization in terms of the perfect JNWsystems. We consider the characterization of the problem under the assumption that the algebra is of monic product type. We have the following theorem.

\section{Theorem 9.3.}

(T3) If $A$ is an alternative algebra, then we have a perfect JNW-system.

(T4) Moreover, if $A$ is an alternative algebra, then the related perfect $J N W$ system is self dual. Hence, $A$ is either the quaternion algebra or the octonion algebra.

Remark 9.4. Originally the authors formulated Theorem 9.3 for a composition algebra. However, by the results of Guy Roos [17] we may suppose that $A$ is an alternative algebra. Precisely, in [17, p. 162], a relation is shown for an algebra $A$ with a unit element $e$ and an involutive anti-automorphism $a \mapsto a^{*}$ such that $a+a^{*}$ and $a a^{*}$ are spanned by $e$ for all $a$ in $A$. With the notation $n(a)=a a^{*}$, if $n$ is a nonsingular mapping into the field of $A$ and $A$ is alternative, then $(A, n)$ is a composition algebra [1].

Proof of (T3). We notice that we have the following identities from the assumption that $A$ is an alternative algebra:

$$
\begin{aligned}
& X(Y X)=(X Y) X, \\
& (X X) Y=X(X Y), \\
& X(Y Y)=(X Y) Y,
\end{aligned}
$$

for every $X, Y \in A$.

Then, letting $X=\sum \alpha_{i} e_{i}$ and $Y=\sum \beta_{j} e_{j}$, we find the following equalities from, respectively, (9.1), (9.2), and (9.3):

$$
\begin{aligned}
& \left(e_{i}, e_{j}: e_{k}\right)+\left(e_{k}, e_{j}: e_{i}\right)=\left(e_{i}: e_{j}, e_{k}\right), \\
& \left(e_{i}, e_{j}: e_{k}\right)+\left(e_{j}, e_{i}: e_{k}\right)=\left(e_{i}: e_{j}, e_{k}\right)+\left(e_{j}: e_{i}, e_{k}\right), \\
& \left(e_{i}, e_{j}: e_{k}\right)+\left(e_{i}, e_{k}: e_{j}\right)=\left(e_{i}, e_{j}: e_{k}\right)+\left(e_{i}: e_{k}, e_{j}\right) .
\end{aligned}
$$

From these equalities we can see the symmetry condition for elements and conclude that our JNW-system is perfect.

Proof of (T4). Here we only show that we can find elements of the sedenion algebra $S=\mathbb{R}\left[1, e_{1}, \ldots, e_{15}\right]$ which do not satisfy the condition of composition algebras. We choose two systems: $\left(e_{8}, e_{10}, e_{2}\right)$ and $\left(e_{12}, e_{14}, e_{2}\right)$. Choosing 
$X=e_{3}+e_{10}$ and $Y=e_{6}-e_{15}$, we can see that $X Y=0$. Here we have chosen the following commutation relations:

$$
e_{3} e_{6}=e_{5}, \quad e_{10} e_{6}=e_{12}, \quad e_{3} e_{15}=e_{12}, \quad e_{10} e_{15}=e_{5} .
$$

Thus, we can see that the condition may not be satisfied for non-self dual perfect systems. In order to prove the assertion, we realize the system by use of the realization theorem, reduce the problem to sedenions, and have to prove it. The proof follows from the relations (9.7) and the table of Example 5.

\section{Acknowledgments}

Grant of the University of Łódź no. 505/692 is acknowledged. The authors are deeply indebted to the referees for improving the text.

\section{References}

[1] R. Abłamowicz, Private communication (2012).

[2] F. L. Castillo Alvarado, J. Lawrynowicz, M. Nowak-Kȩpczyk, Fractals Modelling of Alloy Thin Films. Temperatures Characteristic for Local Phase Transitions. In Applied Complex and Quaternionic Approximation, Ralitza K. Kovaczeva, J. Ławrynowicz, and S. Marchiafava (Eds.), Ediz. Nuova Cultura Univ. "La Sapienza", Roma, 2009, 207-236.

[3] E. Ising, Beitrag zur Theorie des Ferromagnetismus. Zschr. f. Phys. 31 (1925), $253-258$

[4] P. Jordan, Über eine Klasse nichtassoziativer hyperkomplexer Algebren. Nachr. d. Ges. d. Wiss. Göttingen 1932, 569-575.

[5] P. Jordan, Über Verallgemeinerungsmöglichkeiten des Formalismus der Quantenmechanik. Nachr. d. Ges. d. Wiss. Göttingen 1933, 209-217.

[6] P. Jordan, Quantenlogik und das kommutative Gesetz. In The Axiomatic Method with Special Reference to Geometry and Physics. L. Henkin, P. Suppes, and A. Tarski, (Eds.), North Holland, Amsterdam, 1959, 365-375.

[7] P. Jordan, J. von Neumann, and E. Wigner, On an Algebraic Generalization of the Quantum Mechanical Formalism. Ann. of Math. 35 (1934), 29-64.

[8] J. Kigami, Analysis on Factals. Cambridge Tracts in Mathematics 143, Cambridge Univ. Press, Cambridge, 2001.

[9] R. Kikuchi, A Theory of Cooperative Phenomena. Phys. Rev. 81 (1951), 9881003.

[10] J. Ławrynowicz, S. Marchiafava, and M. Nowak-Kępczyk, Periodicity Theorem for Structure Fractals in Quaternionic Formulation. Internat. J. of Geom. Meth. in Modern Phys. 3 (2006), 1167-1197.

[11] J. Ławrynowicz, S. Marchiafava, and A. Niemczynowicz, An Approach to Models of Order-Disorder and Ising Lattices. Adv. Appl. Clifford Alg. 20 (2010), $733-743$.

[12] J. Ławrynowicz, O. Suzuki, and A. Niemczynowicz, Fractals and Chaos Related to Ising-Onsager-Zhang Lattices vs. the Jordan-von Neumann-Wigner Procedures. Ternary Approach. To appear. 
[13] J. Eawrynowicz, M. Nowak-Kȩpczyk, and O. Suzuki, A Duality Theorem for Inoculated Graded Fractal Bundles vs. Cuntz Algebras and Their Central Extensions. Internat. J. of Pure and Appl. Math. 52 (2009), 315-337.

[14] J. Ławrynowicz, M. Nowak-Kȩpczyk, and O. Suzuki, Fractals and Chaos Related to Ising-Onsager-Zhang Lattices vs. the Jordan-von Neumann-Wigner Procedures. Quaternary Approach. Internat. J. of Bifurcations and Chaos, 22, No. 1 (2012) 1230003 (19 pages) (http://www.ee.cityu.edu.hk/rgchen/ iJBC/iJBC_accepted.htm).

[15] J. Lawrynowicz and O. Suzuki, Periodicity Theorems for Graded Fractal Bundles Related to Clifford Structures. Internat. J. Theor. Phys. 40 (2001), 387-397.

[16] L. Onsager, Crystal Statistics I. A Two-Dimensional Model with an OrderDisorder Transition. Phys. Rev. 65 (1944), 117-149.

[17] G. Roos, Exceptional Symmetric Domains. 1: Cayley Algebras. In Symmetries in Complex Analysis, B. Gilligan and G. Roos, 468, Contemporary Mathematics, American Mathematical Society (2008).

[18] G. S. Staples, Clifford Algebras, Graph Problems, and Computational Complexity. The 8th Internat. Conf. in Clifford Algebras, Campinas, Brazil, 2008; with O. Schott, Reductions in Computational Complexity Using Clifford Algebras. Adv. Appl. Clifford Alg. 20 (2010), 121-140.

[19] Y. Uetani and O. Suzuki, A Method of Formal Language for Knot Theory (I). Realization Problem of Knots by Knot Sequences. Bull. Soc. Sci. Letters Łódź Sér. Rech. Déform. 60 No. 3 (2010), 9-26.

[20] Z-D. Zhang, Conjectures on Exact Solution of Three-dimensional (3D) Simple Orthorhombic Ising Lattice. Responses to Comment on a Recent Conjectured Solution of the Three-Dimensional Ising Model [by F.-Y. Wu, B. M. McCoy, M. E. Fisher, and L. Chayes]; Response to Comment on Conjectures on Exact Solution of Three-Dimensional (3D) Simple Orthorhombic Ising Lattices [by J. H. H. Perk], Philosophical Magazine 87 (2007), 5309-5419; 88 (2007), 30973101; 89 (2007), 765-768.

[21] Z-D. Zhang and N. H. March, Conformal Invariance in the Three-Dimensional (3D) Ising Model and Quaternionic Hilbert Space. (Oct. 2011, 16 pp.) (http://arxiv.org/abs/1110.5527).

\author{
J. Ławrynowicz \\ Institute of Physics, University of Łódź \\ Pomorska 149/153, PL-90-236 Łódź \\ Poland \\ Institute of Mathematics, Polish Academy of Sciences \\ Łódź Branch, Banacha 22, PL-90-238 Łódź, Poland \\ e-mail: jlawryno@uni.lodz.pl \\ O. Suzuki \\ Department of Computer and System Analysis \\ College of Humanities and Sciences, Nihon University \\ Sakurajosui 3-25-40 \\ 156-8550 Setagaya-ku, Tokyo \\ Japan \\ e-mail: osuzuki@cssa.chs.nihon-u.ac.jp
}



A. Niemczynowicz
Department of Relativity Physics
University of Warmia and Mazury
Słoneczna 54
PL-10-710 Olsztyn
Poland
e-mail: niemaga@matman.uwm.edu.pl

Received: November 1, 2011.

Accepted: May 1, 2012.

Open Access This article is distributed under the terms of the Creative Commons Attribution License which permits any use, distribution, and reproduction in any medium, provided the original author(s) and the source are credited. 